also den commutirten Ausschlag:

$$
\varphi_{1}-\varphi_{2}=2 \alpha E F \text {. }
$$

Wie man bei Doppelschaltung die Contactpotentialdifferenzen berücksichtigt, hat $\mathrm{Hallwachs}^{1}$ ) gezeigt.

Aus obenstehenden Gleichungen ersieht man, dass man $r_{23}$ bis auf $1 / 3000$ Volt bestimmen kann, am einfachsten aus:

$$
\varphi_{1}+\varphi_{2}: \varphi_{1}-\varphi_{2}=r_{12}: E,
$$

wo das bekannte Potential $E$ etwa durch ein Calomelelement geliefert wird. Das bietet uns die Möglichkeit, die Contactpotentialdifferenzen verschiedener Metalle zu messen. Man hat zu diesem Zwecke aus jedem der beiden zu untersuchenden Metalle ein Quadrantenpaar anzufertigen, diese statt der bisherigen in den Apparat einzusetzen und die Grösse $r_{23} \mathbf{z u}$ bestimmen; dies ist die gesuchte Contactpotentialdifferenz der beiden Metalle.

Königsberg i. Pr., Mathematisch-physikalisches Laboratorium, im Sommer 1888.

\title{
VIII. Ueber die katalytische Wirkung der Metalle auf Knallgas und die Occlusion des Wasserstoffs ${ }^{2}$; von Alfred Berliner. \\ (Hierza Taf. Vl Fig. $7-8$. )
}

$\S 1$. Berzelius ${ }^{3}$ ) bezeichnete die Kraft gewisser Körper, andere umzaändern oder zu zersetzen, ohne sich mit ihnen oder einem ihrer Bestandtheile zu verbinden, mit dem Namen ,katalytische Kraft", und die dadurch veranlasste Reaction mit dem Namen „Katalyse“. Eine katalytische Wirkung übt z. B. glühendes $\mathrm{Kupfer}^{4}$ ) auf Ammoniakgas aus, wodurch dieses in seine Elemente zerlegt wird, ohne dass das Kupfer hierdurch eine Veränderung erleidet; eine weitere katalytische

1) Hallwachs, Wied. Ann. 29. p. 5. 1886.

2) Inauguraldissertation, für die Annalen gekürzt vom Hern Verfasser.

3) Berzelius, Ann. de chim. et de phys. 37. p. 66. 1836.

4) Mitscherlich, Pogg. Ann. 55. p. 209.1842. 
Wirkung ist diejenige der Metalle auf Knallgas, wodurch dieses in Wasser verwandelt wird, und schliesslich, um noch einige Beispiele für die Katalyse einer Flüssigkeit anzuführen, sei die Umsetzung ${ }^{1}$ ) des Rohrzuckers durch Schwefelsäure in Invertzucker erwähnt und ebenso die Umwandlung von Alkohol durch Schwefelsäure in Aether.

Neuerdings hat man jedoch für viele Fälle erkannt, dass die die Katalyse bedingenden Körper eine Veränderung erleiden, indem sie ein Zwischenproduct bilden, welches jedoch stets wieder in seine Bestandtheile zerfällt. So bildet sich bei der Umsetzung des Alkohols in Aether durch Schwefelsäure zuerst Aethylschwefelsäure, welche sich mit einem zweiten Molecül Alkohol in Aether und freie Schwefelsäure wieder zersetzt, sodass die angewandte Menge stets unverändert bleibt und unbegrenzte Mengen Alkohols zersetzen kann. Es ist hiernach nicht unwahrscheinlich, dass sämmtliche Fälle katalytischer Wirkung auf Bildung solcher Zwischenproducte beruhen, auch diejenigen Fälle, bei welchen das Zwischenproduct noch gar nicht oder doch nicht mit Sicherheit nachgewiesen werden konnte. Auch mit dieser veränderten Ansicht über die Katalyse ist folgende Definition verträglich: „Ein Stoff, welcher zu zwei oder mehreren anderen hinzugebracht, unbegrenzte Mengen derselben zu chemischer Verbindung bringt, ohne selbst dabei eine fortschreitende Veränderung zu erleiden, wird ein ,katalytisches Agens" und die dadurch veranlasste Reaction "Katalyse" genannt."

Die folgende Arbeit soll sich mit der katalytischen Wirkung der Metalle auf Knallgas beschäftigen. Ueber die Katalyse des Knallgases liegen bereits Untersuchungen von Dulong und Thénard ${ }^{2}$ ), Faraday ${ }^{3}$ ), Heury ${ }^{1}$ ), 'Turner ${ }^{5}$ ) und Loew ${ }^{6}$ ) vor.

1) Mitscherlich, l. e. p. 210.

2) Dulong u. Thénard, Anu. der Phys. 76. p. 81. 1824.

3) Faraday, Pogg. Anu. 33. p. 149. 1834.

4) Henry, Phil. Mag. (3) 6. p. 354. 1835.

5) Turner, Lieb. Anin. 2. p. 210. 1824.

6) O. Loew, Journ. f. prakt. Chem. (2) 11. p. 372.1875. 
Nach Dulong unà Thénard bewirken alle Metalle und einige Erden die chemische Vereinigung von Sauerstoff und Wasserstoff zu Wasser, und zwar Platin, Palladium, Rhodium und Iridium bei Zimmertemperatur, andere erst bei erhöhter, alle jedoch bei Temperaturen bis zum Siedepunkt des Quecksilbers. Faraday fand, dass Platin die chemische Vereinigung des Knallgases zu Wasser bei Zimmertemperatur nur dann bewirkt, wenn es eine absolut reine metallische Oberfläche hat.

Turner und Henry untersuchten die Wirkung des Platins auf verschiedene Grasgemische, letzterer ausserdem die katalytische Wirkung der Drehspäne von Kupfer und Eisen, sowie die Wirkung der Zinkfolie und der Holzkohle, und fand, dass diese Substanzen erst bei einer Temperatur, die nahe dem Siedepunkt des Quecksilbers liegt, auf Knallgas einwirken.

Loew hat lediglich das Glas zum Gegenstande seiner Lintersuchungen gemacht und fand, dass dieses bei einer Temperatur, die nahe dem Siedepunkt des Quecksilbers liegt, katalytisch zu wirken beginnt.

§ 2. Auf Veranlassung von Hrn. Prof. Warburg habe ich es unternommen, die Versuche über die Katalyse des Knallgases zu wiederholen und zu erweitern. Für den schon von Berthelot') ausgesprochenen Zusammenhang mit der Wasserstoffocclusion ergaben sich dabei neue Belege, und es schlossen sich so an die Versuche über die Katalyse solche über die Wasserstoffocclusion an.

Die von Faraday angegebenen Versuche wurden nachgemacht und die angegebenen Resultate vollständig bestätigt gefunden. Als die Faraday'schen Versuche modificirt wurden, zeigte sich sehr bald, dass die Platinbleche, selbst wenn ihre Oberfläche immer gleichmässig gereinigt war, was dadurch erreicht wurde, dass sie ca. fünf Minuten als positive Electrode in verdünnter Schwefelsäure gebraucht und hierauf in destillirtem Wasser gut abgespült wurden, keineswegs gleich stark auf Knallgas einwirkten. Einzelne Bleche be-

1) Berthelot, Compt. rend. 84. p. 1383, 1882. 
wirkten die chemische Verbindung des Knallgases, sobald sie in das Gasgemisch gebracht wurden, augenblicklich und führten eine Explosion des Knallgases herbei; andere fingen sofort an, langsam aber gleichmässig zu wirken, d. h. sie bewirkten in gleichen Zeiten die chemische Verbindung von gleichen Knallgasmengen; bei wieder anderen verstrichen einige Minuten, ehe sie zu wirken anfingen, und endlich waren manche überhaupt nicht fähig, die katalytische Wirkung hervorzubringen. Diese Art der Vorbereitung war also jedenfalls von sehr vielen Zufälligkeiten abhängig, die nicht leicht zu umgehen waren, und deshalb versuchte ich, die katalytische Wirkung des Platins ohne jede vorherige Vorbereitung lediglich durch Temperaturerhöhung vorzubringen, wie es Dulong und Thénard mit anderen Metallen gemacht.

§ 3. Da letztere nicht angegeben haben, wie sie ihre Versuche anstellten, will ich die Methode angeben, die bei meinen Versuchen in Anwendung kam. Das auf Katalyse hin zu untersuchende Metall in Blechform oder als Pulver, wie es mir gerade zur Verfügung stand, wurde ohne jedwede vorherige Vorbereitung - wenn die Oberfläche der Bleche sichtbar verunreinigt war, so wurde dieselbe mit Fliesspapier und Alkohol gereinigt - in ein Glasrohr gebracht und durch dasselbe ein constanter Strom von electrolytischem Knallgase ${ }^{1}$ ) geleitet. Wurde das in einer bestimmten Zeit eintretende Gas und ebenso das austretende seinem Volumen nach gemessen, so ergab die Differenz der beiden die Grösse der katalytischen Wirkung.

Der zur Verwendung gekommene Apparat war so eingerichtet, wie es Fig. 7 zeigt.

Der Wasserzersetzungsapparat $A$, zu welchem den galvanischen Strom fünf Siemens'sche sogenannte ,amerikani-

1) Es wurden auch Versuche mit einem Gasgemisch, wie es der Bunsen'sche Brenner liefert, und ebenso mit Wasserstoff und Luft angestellt. Allein diese Gase haben den Nachtheil, dass eine gleichmässige Mischung nicht ohne Schwierigkeiten zu erzielen ist, und durch die Verschiedenheit in der Zusammensetzung der Gase die Beobachtungen sehr erschwert werden. 
sche" Elemente lieferten, erzeugte das Knallgas. Durch Einschaltung eines regulirbaren Widerstandes wurde die an einer Tangentenbussole beobachtete Stromstärke stets auf demselben Werth gehalten, sodass die Menge des in jeder Minute erzeugten Knallgases während der Dauer eines Versuches constant blieb. Dasselbe ging durch die Rohrleitung $a$ zu dem Blasenzähler $B_{1}$. Derselbe war ebenso wie der Blasenzähler $B_{2}$ aus einem Reagensglase gebildet, welches halb mit Wasser gefüllt und mit einem doppelt durchbohrten Kork verschlossen war, durch welchen die in beiden Apparaten gleich weiten Glasröhren gingen. Aus dem Blasenzähler $B_{1}$ wurde das $G a s$ in den Verdichtungsapparat $C$ geleitet. Derselbe bestand aus der Glasröhre $a$, von $1,5 \mathrm{~cm}$ lichter Weite und $10 \mathrm{~cm}$ Länge, die am unteren Ende zugeschmolzen war. Am oberen Ende waren zwei rechtwinklig gebogene Glasrohre angesetzt, von denen das bei $b$, durch welches das Gas einströmte, nach oben verlängert war und durch die Oeffnung $d$ gestattete, die zu untersuschende Substanz in den Apparat zu bringen. Die Oeffnung $d$ konnte durch einen Gummipfropfen verschlossen werden. Die zweite Röhre $c$, durch welche das Gas wieder ausströmte, ging bis auf den Boden des Rohres a. Der ganze Apparat steckte auf ca. $12 \mathrm{~cm}$ in dem Luftbade $D$, dessen Temperatur durch einen Reichert'schen Thermostaten $F$ regulirt wurde. $G$ war ein mit Stickstoff' gefülltes Quecksilberthermometer, welches Theilung bis $450^{\circ} \mathrm{C}$. hatte. Da die Temperatur in dem Luftbade mit der Höhe variirte, wurde das Thermometer so weit einge. senkt, dass die Quecksilberkugel in gleicher Höhe mit dem Boden des Verdichtungsgefässes war und ganz an diesem anlag. Das bei $e$ austretende Gas ging in die Kühlschlange $E$, die durch ein $150 \mathrm{~cm}$ langes Glasrohr gebildet war und auf dem Experimentirtische lag. Die Anwendung dieser Kühlschlange wurde nöthig, um das aus dem Verdichtungsapparate austretende heisse Gas vor seinem Eintritt in den Blasenzähler $B_{2}$ wieder auf die Temperatur des einströmenden Gases, also a uf Zimmertemperatur abzukühlen, damit das durch beide Blasenzähler zugleich hindurchgehende Gasquantum in beiden Zählern gleich viele Blasen bildete. Das aus 
dem Blasenzähler $B_{2}$ austretende Gas wurde in das Freie geleitet.

$\S$ 4. Die Versuche selbst wurden folgendermassen angestellt. Nachdem der Verdichtungsapparat sorgfältig gereinigt und getrocknet in das Luftbad gebracht und alle Schlauchverbindungen mit den übrigen Apparaten hergestellt waren, wurde der galvanische Strom geschlossen, circa $1 / 2$ Stunde Knallgas durch den Apparat geleitet und hierbei die Gleichmässigkeit der Blasen in den beiden Blasenzählern regulirt. Dieselben waren an einem eisernen Stativ nebeneinander angebracht, und während $B_{1}$ stets unverändert blieb, wurde $B_{2}$ so lange geneigt, bis vollständige Gleichmässigkeit in beiden Blasenzählern herrschte. Hierauf wurde das Lufthad erwärmt, die Folge daron war, dass die Blasen sofort unregelmässig kamen, und zwar gingen bei einer Geschwindigkeit von 40 Blasen in der Minute durch den ersten Blasenzähler in derselben Zeit 44 durch den zweiten. Wurde aber die Temperatur in irgend einer Höhe constant erhalten, so schwand bald die Verschiedenheit, und nach wenigen Minuten arbeiteten die Blasenzähler wieder vollständig gleichmässig. Hierbei wurde die Temperatur bis circa $430^{\circ} \mathrm{C}$. gesteigert und dadurch die Gewissheit erlangt, dass der leere Apparat, also die Glaswände, bei dieser Temperatur das Knallgas nicht chemisch veränderten. Sodann wurde das Luftbad wieder abgekühlt und bei gewöhnlicher Temperatur die Gleichheit der Blasen controlirt. Diese Erwärmung des leeren Apparates fand vor jedem neuen Versuche statt. Bei Zimmertemperatur wurde dann der Gummipfropfen bei $d$ herausgezogen und die zu untersuchende Substanz schnell in den Apparat gebracht, worauf dieser sofort wieder geschlossen wurde. Da in dieser Zeit etwas Knallgas entweicht, wurde nach dem Einbringen der Substanz circa 10 Minuten lang Knallgas durch den Apparat geleitet, ohne eine Bobachtung anzustellen, um ganz sicher zu sein, dass reines Knaligas den Verdichtungsapparat erfülle. Nachdem auch jetzt wieder die Gleichmässigkeit der Blasen festgestellt war, wurde mit dem Erwärmen des Luftbades begonnen. Von circa $20^{\circ}$ zu $20^{\circ}$ wurde die Temperatur constant enthalten, 
was durch gieichmässiges Verstellen der Schraube am Thermostaten sehr leicht erreicht werden kann, und die Blasen wieder gezählt. Sobald im zweiten Blasenzähler die Blasen langsamer kamen als im ersten und somit der Anfang der katalytischen Wirkung angezeigt war, wurde die Temperatur längere Zeit auf gleicher Höhe gehalten, um zu sehen, ob die Verdichtung mit der Zeit zunähme. Ebenso wurde bei höheren Temperaturen verfahren, wenn die Wasserbildung bereits ziemlich stark vor sich ging. Nachdem die Temperatur bis circa $350^{\circ} \mathrm{C}$. gestiegen war, wurde ebenso stufenweise wieder abgekühlt. Dadurch dass von einer bestimmten Temperatur an die Blasen wieder gleichmässig durch beide Blasenzähler hindurchgingen, war der Beweis geliefert, dass die Verschiedenheit in den Blasen lediglich durch die Katalyse hervorgerufen war und nicht etwa durch einen Fehler im Apparate. Während des Versuches wurden alle $15 \mathrm{Mi}$ nuten die Anzahl der während einer Minute einströmenden Blasen gezählt und eventuell durch Stromregulirung auf gleicher Höhe gehalten. Die Grösse der Blasen wurde von Zeit zu Zeit dadurch gemessen, dass 200-500 Blasen in einem graduirten Rohre aufgefangen wurden; die Grösse derselben wurde mit sehr kleinen Unterschieden immer gleich gefunden. Eine Reduction derselben auf $0^{0}$ und $760 \mathrm{~mm}$ Druck fand nicht statt, und gilt als Mittel $20^{\circ} \mathrm{C}$. und $740 \mathrm{~mm}$ Druck.

§5. Die Resultate, welche bei diesen Versuchen erhalten wurden, sind der leichteren Uebersichtlichkeit wegen in Tabellen wiedergegeben, und zwar ist in der ersten Rubrik angegeben, welche Zeit in Stunden und Minuten seit Beginn des Versuches vergangen, in der zweiten die Temperatur des Luftbades, in der dritten und vierten die Anzahl der ein- und austretenden B!asen und in der letzten, wie viel Cubikeentimeter Knallgas in der Minute zu Wasser verdichtet wurde. Als Beispiel möge hier ein Theil der ersten so erhaltenen Tabelle angeführt werden, welche sich auf Platin bezieht. Die anderen Tabellen finden sich in meiner Dissertation. 
Tabelle I.

Platin, in Blechform ca. $1 / 35 \mathrm{~mm}$ stark $84 \mathrm{ing}$.

\begin{tabular}{|c|c|c|c|c|c|}
\hline \multirow{2}{*}{$\begin{array}{c}\text { Verflossene } \\
\text { Zeit seit } \\
\text { Beginn } \\
\text { des Vers. }\end{array}$} & \multirow{2}{*}{ Temp } & \multicolumn{2}{|c|}{$\begin{array}{l}\text { Zahl der } \\
\text { Blasen }\end{array}$} & \multirow{2}{*}{$\begin{array}{l}\text { Cubik- } \\
\text { centimet. } \\
\text { Verdicht. }\end{array}$} & \multirow{2}{*}{ Bemerkungen. } \\
\hline & & einstr. & ausstr. & & \\
\hline$\ldots$ h $15^{\mathrm{m}}$ & $162^{\circ}$ & 50 & 50 & 0 & In der Minute gehe \\
\hline-47 & 181 & 50 & 50 & 0 & 44 Blasen durch de \\
\hline-53 & 213 & 5 & 4 & 0,9 & Blasenzähler $\mathbf{I}, \quad$ vo \\
\hline 18 & 221 & 7 & 5 & 1,3 & denen jede $102,5 \mathrm{cmm}$ \\
\hline 130 & 236 & 7 & 3 & 2,57 & Volumen hat. \\
\hline
\end{tabular}

Der Versuch wird unterbrochen; derselbe wird mit dem. selben Platin wiederholt, dem noch $73 \mathrm{mg}$ Platin zugesetzt werden, i. G. also $157 \mathrm{mg}$ von $1 / 35$ und $1 / 36 \mathrm{~mm}$ starkem Blech.

\begin{tabular}{ll|l|r|r|l|}
$\overline{-}^{\mathrm{h}}$ & $21^{\mathrm{m}}$ & $187^{0}$ & 12 & 11 & 0,34 \\
- & 26 & 190 & 8 & 7 & 0,54 \\
- & 38 & 190 & 8 & 7 & 0,54 \\
- & 49 & 210 & 4 & 3 & 1,19 \\
1 & 10 & 227 & 15 & 4 & 3,3 \\
1 & 18 & 242 & 34 & 5 & 3,83 \\
1 & 30 & 242 & 34 & 5 & 3,83 \\
1 & 38 & 249 & 52 & 6 & 3,97 \\
1 & 57 & 212 & 2 & 1 & 2,25 \\
2 & 5 & 213 & 8 & 5 & 2,57 \\
2 & 12 & 206 & 3 & 2 & 1,49 \\
2 & 44 & 175 & 7 & 6 & 0,63
\end{tabular}

$\S 6$. Aus den Tabellen geht hervor, dass die kata. lytische Wirkung bei den verschiedenen Substanzen bei ganz bestimmten Temperaturen beginnt und mit Steigerung der Temperatur zunimmt, und zwar liegt diese Anfangstemperatur für Platinschwamm unterhalb der Zimmertemperatur, für Platin in Blechform und ebenso für platinirtes Platin. blech bei $180-190^{\circ}$ C., für Palladium in Blechform bei ca. $175^{\circ}$ C., für fein vertheiltes Silber bei ca. $265^{\circ}$ C., für ferrum limatum bei ca. $270^{\circ} \mathrm{C}$, für Kohle bei ca. $275^{\circ} \mathrm{C}$. und endlich für Kupfer bei ca. $280^{\circ} \mathrm{C}$.

$\S 7$. Zink und Aluminium zeigten keine katalytische Wirkung, obgleich ersteres bis $350^{\circ}$, letzteres bis $440^{\circ} \mathrm{C}$. erwärmt wurde. Ebenso wenig gelang es, bei Glaspulver eine katalytische Wirkung zu constatiren. Weiter schei- 
nen die Versuche auch zu beweisen, dass die katalytische Wirkung lediglich von der Temperatur und nicht von der Dauer der Temperatureinwirkung abhängig ist, denn die Menge des in der Minute zu Wasser verbundenen Knallgases blieb bei constanter Temperatur auch annähernd constant, nahm jedoch sofort zu, sobald die Temperatur erhöht wurde. Wurde andererseits abgekühlt, so nahm auch sofort wieder die katalytische Wirkung ab, und mehrere Versuche zeigten bei genügend langsamer Abkühlung als Endtemperatur der Katalyse ungefähr dieselbe, als die Anfungstemperatur.

§. Ein Versuch, sämmtliche Substanzen, welche die Katalyse zeigen, ihrer Wirkung nach in eine Reihe zu bringen, dürfte nicht gelingen, da die Grösse der katalytischen Wirkung derselben Substanz je nach der Form eine ganz verschiedene ist. Mehrere Versuche haben ergeben, dass die katalytische Wirkung einer Substanz um so grösser ist, je feiner zerteilt dieselbe in Anwendung kommt. Aber es ist nicht nur die grössere Oberfäche, welche einem fein zertheilten Körper die grössere katalytische Wirkung ertheilt; denn während ein ca. $1,5 \mathrm{~mm}$ dicker Platindraht die Katalyse nur in ganz minimaler Weise zeigte, wirkte ein Platinblech von der Dicke von $1 / 4 \mathrm{~mm}$ bei gleicher Oberfläche sebr stark. Wahrscheinlich kommt es auf das Verhältniss von Oberfläche zu Volumen an. Bezeichnen wir bei einem Blech die Länge mit $l$, die Breite mit $b$ und die Dicke mit $d$, und entsprechend bei einem Draht die Länge mit $l$ und den Radius mit $r$, so erhält man:

für „ein Blech“:

$\begin{aligned} \text { Oberflïche }=O & =2 b l \\ \text { Volumen }=V & =b l d \\ O / V & =2 / d\end{aligned}$

für einen Draht:

Obertäche $=O=2 r \pi l$

Volumen $=\frac{\Gamma=r^{3} \pi l}{O / V=2 / \boldsymbol{r}}$. 
Im vorliegenden Falle ist dieses Verhältniss $O / V$ in Bezug auf Millimeter für den Draht $=\frac{8}{3}$, für das Blech $={ }^{96} / \mathrm{x}, \mathrm{d}$. h. für das Blech ist dieses Verhältniss 36 mal so gross als für den Draht; wird also an der Oberfläche des Metalls eine bestimmte Quantität Knallgas zur chemischen Verbindung gebracht, so wird die dabei eintretende Temperaturerhöhung um so grösser sein, je geringer die Masse des Metalls ist, je weniger Wärme also durch das Metall abgeleitet wird; da nun Temperaturerhöhung die katalytische Wirkung begünstigt, wie aus den Versuchen hervorgeht, so wird ein dünnes Blech viel stärker katalytisch wirken, als ein dicker Draht mit gleicher Obertiäche.

§ 9. Ueberblickt man diese Versuche, so scheint sich eine Beziehung zu ergeben zwischen der katalytischen Wirkung der Metalle auf Knallgas und ihrer Fähigkeit, Wasserstoff zu occludiren. Eine solche Beziehung ist gewiss schon von vielen, die sich mit dieser katalytischen Wirkung beschäftigt haben, angenommen, von Berthelot bestimmter behauptet worden.

Nach Versuchen von Graha ${ }^{1}$ ) occludiren Palladium, Platin, Platinschwamm und Platinmohr eine ungleich grössere Menge Wasserstoff als alle anderen Metalle, und gerade diese Substanzen sind es auch, welche sich durch ihre katalytische Wirkung ganz besonders auszeichnen. Andererseits occludiren Zink und Aluminium keine merklichen Mengen Wasserstoff, und gerade diese Substanzen zeigten bei meinen Versuchen keine katalytische Wirkung oder doch nur eine so geringe, dass sie durch die Methode, die ich bei meinen Versuchen anwandte, nicht nachweisbar war.

Dabei erscheint es zunächst auffällig, dass Palladium, welches nach Berthelot ${ }^{2}$ 7-12 mal so viel Wasserstoff occludirt als Platin, nach Graham ${ }^{3}$ ) sogar $100-130$ mal so viel, keine erheblich grössere Wirkung zeigte als dieses. Jedenfalls erschien es angezeigt, die Beziehung zwischen katalytischer Wirkung und Occlusion von Wasserstoff weiter

1) Graham, Pogg. Ann. 129. p. 549. 1866.

2) Berthelot, Compt. rend. 94. p. 1383. 1882.

3) Graham, Phil. Mag. (4) 36. 63. 1. 1868. 139. 
zu verfolgen. Die vorliegenden Angaben über die Gasocclussion der Metalle und speciell des Platins sind nur wenige, und diese differiren untereinander sehr. Nach den Angaben von Graham occludirt Platin bei Rothgluth das 5 -6fache seines eigenen Volumens an Wasserstoff, nach Berthelot gibt es zwei Wasserstoffverbindungen des Platins mit dem 80- und 120 fachen Volumen Wasserstoff. Die Angaben üher die Sauerstoffocclusion sind ebenso verschieden, nach (Grahamin) findet gar keine statt.

Ich habe mich deshalb entschlossen, eigene Versuche über die Occlussion des Wasserstoffs durch Platin und Palladium anzustellen; dabei wurden die Versuche auch auf andere Gase ausgedehnt. Das Folgende enthält die Ergebnisse dieser Untersuchungen.

$\$ 10$. Um die allgemeinen Bedingungen kennen zu lernen, welche die Occlusion begünstigen, und welche ihr hinderlich sind, wurden zuerst Versuche mit Palladium angestellt, da dieses Metall am meisten Wasserstoff zu occludiren vermag und infolge dessen auch eine $W$ asserstoffocclusion, resp. das Ausbleiben derselven am leichtesten erkennen lässt.

Der Nachweis der Occlusion wurde so geführt, dass das Paladium mit einem abgemessenen Volumen Wasserstoff zusammengebracht und bei constantem Druck und constanter Temperatur direct die Volumenabnahme gemessen wurde. Dem Metall wurde vor jeder neuen Beladung das occludirte Gas durch Ausglühen im Vacuum mittelst des galvanischen Stromes entzogen und dieses ebenfalls dem Volumen nach gemessen, sodass durch die Differenz der beiden Gasvolumina zugleich bestimmt wurde, wie viel Gas im Vacuum bei Zimmertemperatur frei geworden.

$\$ 11$. Ein diesen Bedingungen entsprechender Apparat ist der in Figur 8 abgebildete.

$A$ ist ein Glasrohr, welches durch den eingeschliffenen Glaspfropfen $B$ verschlossen wurde. Durch diesen letzteren, welcher hohl war, gingen die beiden Glasröhren $a$, durch welche die als Zuleitungsdrähte des galvanischen Stromes

1) Graham, Phil. Mag. (4) 36. 63. 1. 139. 1868. Aun. d. Phys, u. Chom. N. F. XxxV. 
dienenden Platindrähte $b$ hindurchgingen. Bei $c$ waren diese Drähte $b$ so eingeschmolzen, dass nur die kleinen Platinhaken $d$ aus dem Glase herausragten, an welche der zu untersuchende Metallstreifen $e$ angehängt werden konnte. $\mathrm{Um}$ die Fettung des Glasstopfens gegen die ausströmende Hitze des glühenden Metalls $e$ zu schützen, wurde derselbe mit Wasser gekühlt, und zwar derart, dass aus der Wasserleitung ständig Wasser in den Hohlraum des Pfropfens einHoss und bei $f$ wieder ablief. Der Hahn $C$ bildete den Abschluss gegen das graduirte Rohr $D$, welches $5 \mathrm{ccm}$ Inhalt hatte und von $10 \mathrm{zu} 10 \mathrm{cmm}$ getheilt war. Die Erweiterung des Rohres bei $\boldsymbol{E}$ war so gewählt, dass ihr Volumen von dem Hahne $F$ bis zum Anfang der Graduirung $D$ ungefähr dasselbe war wie dasjenige des Rohres $A$. $G$ war ein Quecksilbergefäss mit dem Ansatzrohr $H$, welches dazu diente, den Höhenstand des Quecksilbers im Gefäss leichter bestimmen zu können. $\boldsymbol{G}$ war mit $\boldsymbol{F}$ durch den Schlauch $J$ verbunden. Unterhalb des Hahnes $C$ mündete das Rohrsystem $K$, welches der Hahn $L$ gegen das Occlusionsgefäss, der Hahn $N$ gegen den Gasentwickelungsapparat, der Hahn $O$ gegen die mit Schwefelsäure gefültte Schale $P$, und der Hahn $M$ gegen die Töpler-Hagen'sche Pumpe abschloss. $\mathrm{Um}$ das beim Glühen freiwerdende Gas auffangen zu können, wurde das capillare Ausflussrohr der Pumpe etwas in das Austlussgefäss hineingeführt, und über das erstere ein graduirtes mit Quecksilber gefülltes Rohr gestülpt.

$\S 12$. Die Versuche wurden so angestellt, dass zuerst das Quecksilber aus dem Gefässe $G$ bis in den Hahn $F$ gelassen wurde, sodass dessen Bohrung noch mit Quecksilber angefüllt war. Hieranf wurde, während die Hähne $N$ und $O$ geschlossen blieben, der ganze Apparat möglichst luftleer gepumpt, die Hähne $M$ und $C$ geschlossen und durch Oeffinen des Hahnes $N$ der im Kipp'schen Apparat entwickelte und sorgfältig gereinigte Wasserstoff in den Apparat gelasssen. Darauf wurde der Hahn $N$ wieder geschlossen und durch Oeffinen des Hahnes $O$, dessen Mündung ca. $1 \mathrm{~mm}$ in Schwefelsäure tauchte, ein etwaiger Ueberdruck, der durch den Kipp'schen Apparat entstanden sein konnte, beseitigt. 
Nun wurde durch Schliessen des Hahnes $L$ in dem graduirten Rohre ein ganz bestimmtes Volumen Wasserstoff von Atmosphärendruck abgeschlossen, und dieses diente zu den Occlusionsversuchen. Durch Oeffinen des Hahnes $C$ vertheilte sich das Gas auch über den luftleeren Raum $A$, und stieg infolge dessen beim Oeffnen des Hahnes $F$ das Quecksilber in die Kugel $E$ und stellte sich bei gleichem Niveau in $D$ und $H$ an einer ganz bestimmten Stelle ein, wenn keine Occlusion stattfand. Dieser Punkt wurde dadurch ermittelt, dass der soeben beschriebene Versuch mehrmals angestellt wurde, ohne das zu untersuchende Metall in den Apparat $A$ zu bringen. Fand̀ später Occlusion statt, so stieg das Quecksilber in $D$ und konnte das Volumen des occludirten Gases direct an der Theilung abgelesen werden, wenn nur stets durch Heben von $G$ der Druck im Innern des Apparates constant erhalten wurde. Um den nicht unbeträchtlichen Schwankungen in der Temperatur und dem Barometerstande Rechnung zu tragen, war direct neben dem Apparate ein Barometer und zwei Thermometer mit $1 / 0^{\circ} \mathrm{C}$. Theilung aufgestellt. Zu Beginn und Schluss jedes Versuches wurde Temperatur und Barometerstand abgelesen und die abgelesene Volumenänderung auf Anfangs Druck und Temperatur reducirt. Durch Berechnung und durch Controlversuche wurde ermittelt, dass einer Temperaturänderung von $1^{\circ} \mathrm{C}$. eine Volumenänderung des abgeschlossenen Gases um $116 \mathrm{cmm}$, sowie einer Druckänderung um $1 \mathrm{~mm}$ einer solchen von $42 \mathrm{cmm}$ entsprach.

§ 13. Das Resultat eines derartigen Versuches war folgendes. Ein Palladiumblech 44,5 mg schwer, welches vor dem Verbringen in den Apparat weder geglüht noch mechanisch oberflächlich gereinigt war, occludirte während mehrerer Stunden keinen Wasserstoff.

Als das Metall im Vacuum gegläht hatte, wurden $450 \mathrm{cmm}$ frei, d. h. das 114 fache Volumen des Palladiums. Nachdem jetzt der Apparat zwei Siunden gestanden hatte, wurde Wasserstoff eingelassen, allein eine merkliche Occlusion fand nicht stat.t. Bei näherer Untersuchung des aus den Apparat genommenen Bleches zeigte sich, dass die Ober- 
fläche nicht rein war, was sich dadurch zu erkennen gab, dass das Palladium in Wasser getaucht von diesem nicht benetzt wurde.

Es lag die Vermuthung nahe, dass durch die im Apparate entstehenden Fettdämpfe oder auch durch Fett, welches sich capillar zu dem Metalle hinzieht, diese Verunreinigung bewirkt werde, und um dies zu constatiren, wurde das Blech im Gebläse stark ausgeglïht, sodass es, erkaltet ins Wasser getaucht, vollständig gleichmässig benetzt wurde. Wurde es hierauf in den Apparat gebracht, so genügte ein einstündiges Verweilen, um seine Oberfläche mit einer Fettschicht zu überziehen, sodass es jetzt von Wasser nicht mehr benetzt wurde. Um weiter zu untersuchen, ob die Oberflächenverunreinigung die Occlusionsfähigkeit aufhebe, wurde das Palladium durch Anwendung als positive Electrode in verdünnter Schwefelsäure gereinigt. Nachdem es in Wasser gut abgespült und zwischen Fliesspapier getrocknet war, wurde es möglichst schnell in den Apparat gebracht und sofort Wasserstoff eingelassen. Momentan occludirte das Palladium das 857 fache seines eigenen Volumens an Wasserstoff.

Man kann das Metall auch durch Ausglühen im Gebläse reinigen. Deshalb wurde das Metall abermals aus dem Apparate herausgenommen und im Gebläse stark ausgeglüht. Schnell in den Apparat zurückgebracht, occludirte das Metall das 860 fache seines eigenen Volumens an Wasserstolf. Wurde jedoch mit dem Einlassen von $W$ asserstoff in den Apparat ein bis zwei Stunden gewartet, so fand keine Wasserstoffocclusion mehr statt.

Gleiche Versuche, die mit Platin angestellt wurden, ergaben dieselben Resultate.

$\S 14$. Um die Occlusionsversuche fortsetzen zu können, musste deshalb der Apparat geändert werden. Da bei früheren Versuchen nie eine derartige Störung bemerkt worden war, obgleich in dem angewandten Apparate ebenfalls Hähne, die mit Fett geschmiert waren, vorhanden waren, dieselben jedoch von dem Metall durch eine längere Rohrleitung getrennt waren, so ging ich auf dieses Princip zurück und schnitt den Apparat $A$ (Fig. $S$ ) über dem Hahne $C$ ab. An 
seine Stelle wurde ein ca. 0,5 m langes Biegerohr angesetzt, an dessen Ende ein weiteres Glasrohr angeschmolzen wurde, in dem der zu untersuchende Metallstreifen, um lier jede Fettung zu vermeiden, mit Platindrähten eingeschmolzen war. Mit diesem Apparate, der in Fig. 8 punktirt angegeben, wurden sämmtliche nun folgenden Occlusionsversuche ausgeführt.

\$15. Wie auch früher schon, begann jeder Versuch damit, dass dem Platin das ganze occludirte Gas durch Ausglühen mittelst des galvanischen Stromes entzogen wurde.

Um dies $z u$ erreichen, ist ein viele Stunden lang andauerndes Hellgelb- bis Weissglühen erforderlich. In der folgenden Tabelle sind die gentuen Angaben über den Verlauf eines neunstündigen Glühens gegeben. In der ersten Rubrik ist die seit Anfang des Glühens verstrichene Zeit gegeben, in der zweiten das während dieser Zeit freigewordene Gasvolumen bei $760 \mathrm{~mm}$ Druck in Cubikmillimetern, in der dritten dasselbe Volumen ausgedrückt als Vielfaches des Platinvolumens, in der vierten das in je 20 Minuten abgegebene Gas wieder ausgedrückt als ein Vielfaches des Platinvolumens.

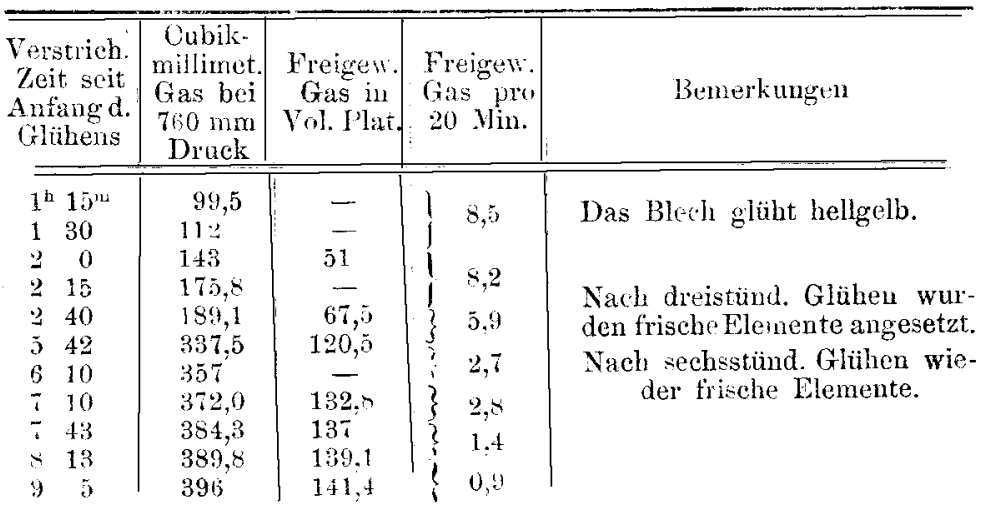

Aus dieser Tabelle ist ersichtlich, dass Platin das occludirte Gas selbst bei sehr hoher Temperatur, Hellgelbglühen, nur äusserst langsam abgibt, und dass selbst nach neunstündigem Glühen noch nicht alles Gas frei geworden war. 
$\S$ 16. Die absoluten Angaben über Occlusionsfähigkeit des Platins differiren deshalb auch sehr stark und sind lediglich daron abhängig, wie lange das dem Occludiren vorhergegangene und nachfolgende Glühen fortgesetzt war. Die Versuche wurden nicht nur mit Wasserstoff, sondern auch mit Kohlenoxydgas, Sauerstoff und Luft angestellt, und zwar in dor Weise, dass das gasfreie Metall mit dem betreffenden Gase zusammengebracht und die directe Volumenverminderung desselben gemessen wurde. Nachher wurde dem Metall durch Glühen das Gas wieder entzogen und dieses ebenfalls dem Volumen nach bestimmt. Die angegebenen Zahlen können jedoch keinen Anspruch auf absolute Genauigkeit machen, da einmal das Volumen des angewandten Metalles sehr klein war, und ausserdem das Volumen der Häkchen, an denen das Metall hing, und welches nur näherungsweise geschätzt werden konnte, ebenfalls mit in Rechnung zu ziehen war und bei dem kleinen Gesammtrolumen schon bedeutende Differenzen hervorrufen konnte. Ein zweiter Fehler ist die Unsicherheit, ob diese Platindrahthäkchen, welche viel schwächer glühten, als das Metall selbst, in derselben Zeit ihr occludirtes Gas abgegeben und sich auch umgekehrt, der grösseren Dicke wegen, durch ihre ganze Masse mit Gas beladen hatten.

\$17. Nur einmal war es möglich, mit demselben Platinblech eine ganze Versuchsreihe anzustellen; d. h. es nacheinander die verschiedenen Gase occludiren zu lassen. Gewöhnlich sprang das Glasrohr, in dem das Metall glühte, oder das Metall schmolz infolge zu starken Glühens durch etc. Die Resultate, welche bei diesen Versuchen erhalten wurden. sind der leichteren Uebersichtlichkeit wegen in der folgenden Tabelle zusammengestellt, und zwar bedeuten die angegebenen Zahlen das Gasvolumen, ausgedrückt als Vielfaches des Platinvolumens. 


\begin{tabular}{|c|c|c|c|c|c|c|c|c|}
\hline & $\begin{array}{c}\text { Beim } \\
\text { Glühen } \\
\text { freigew } \\
\text { Gas }\end{array}$ & $\underset{\mathrm{H}}{\text { Ocelud }}$ & $\begin{array}{c}\text { Beim } \\
\text { Glühen } \\
\text { freigew. } \\
\mathbf{H}\end{array}$ & $\underset{c o}{\text { Occlud. }}$ & \begin{tabular}{|c} 
Durch \\
Glühen \\
freigew. \\
Co
\end{tabular} & $\underset{0}{\text { Oeclnd. }}$ & \begin{tabular}{|c} 
Durch \\
Glühen \\
freigew. \\
Sauer- \\
stoff \\
\end{tabular} & $\begin{array}{l}\text { Oceind } \\
\text { Luft }\end{array}$ \\
\hline & $8 \pi$ & 236 & 170 & - & - & - & - & $\ldots$ \\
\hline Fäk & $\ldots$ & 271 & 148 & 108 & 96 & - & - & - \\
\hline$\pi$ & - & $\ldots$ & - & 90 & 84 & 100 & 57 & 83 \\
\hline Pt $54 \mathrm{mg}+6 \mathrm{mg}$ & & & & & & & & \\
\hline Häkchen . & $i 1$ & 204 & $1+1$ & - & - & - & 一 & - \\
\hline Platin $48,2 \mathrm{mg}+10 \mathrm{mg}$ & & & & & & & & \\
\hline 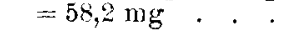 & 85 & - & - & 一 & - & - & - & - \\
\hline Platin $87 \mathrm{mg}+6 \mathrm{mg}$ & 227 & & \multicolumn{5}{|c|}{ (während 251 , stünligen Glühens) } & \\
\hline Pt $80.5 \mathrm{mg}+6,5 \mathrm{mg}$ & - & 127 & 83 & $-\cdots$ & - & - & ... & - \\
\hline
\end{tabular}

Wollen wir aus diesen Tabellen einen Schluss auf die Occlusionsfähigkeit des Platins ziehen, so müssen wir zuerst constatiren, dass jedes Platin in gewöhnlichem Zustande sehr viel Gas occludirt enthält, welches es nur äusserst schwer wieder abgibt. Sieht man von der Zahl 227 ab, so stimmen bei den übrigen Platinsorten die durch Glühen dem Platin entzogenen Gasvolumina ungefähr überein, und zwar betragen dieselben rund das 80 fache des eigenen Platinvolumens. Ausserdem sicht man, dass Platin, welches gasfrei ist, sich ron neuem mit Gas beladen kann, und zwar scheint die chemische Beschaffenheit der Gase, $H$ ausgenommen, wenig Einfluss auszuïben. Die mit Kohlenoxydgas, Sauerstoff und Luft angestellten Versuche ergaben das neu occludirte Gasvolumen verhältnissmässtg wenig verschieden von dem vor der neuen Beladung durch Glühen entzogenen. Wasserstoff wird in viel grösseren Quantitäten occludirt und auch bierbei spielt wieder eine grosse Rolle, ob das Platin vollständig gasfrei den Wasserstoff occludirt oder aber das natürlich occludirte Gas noch enthält. In letzterem Falle, worïber nur ein Versuch vorliegt, occludirte das Metall das 127 fache seines eigenen Volumens Wasserstoff, gab im Vacunm bei Zimmertemperatur das 44 fache Volumen ab und schliesslich beim Glühen das 83 fache. Entzieht man jedoch

1) Bei allen übrigen Versuchen wurde das Metall nur $8-10$ Stunden vor jeder Neubeladung mit Gas geglüht. 
dem Platin alles occludirte Gas, so beträgt das Volumen des occludirten Wasserstoffs über das 200 fache des Platinvolumens, wovon es wieder einen grossen Theil schon bei Zimmertemperatur im Vacuum abgibt.

\$18. Eine gleiche Versuchsreihe wurde mit Palladium angestellt, deren Resultate in der folgenden Tabelle wiedergegeben sind.

\begin{tabular}{|c|c|c|c|c|c|c|}
\hline & $\begin{array}{l}\text { Beim Glü- } \\
\text { hen frei- } \\
\text { gew. Gas }\end{array}$ & $\begin{array}{l}\text { Ocela- } \\
\text { dirter } \mathrm{H}\end{array}$ & $\begin{array}{l}\text { Beim Glü- } \\
\text { hen frei- } \\
\text { gew. H }\end{array}$ & dirter 0 & $\begin{array}{c}\text { Beim Glü- } \\
\text { hen fiei- } \\
\text { gew. } 0\end{array}$ & $\begin{array}{l}\text { Occlud. } \\
\text { Luft }\end{array}$ \\
\hline $\begin{array}{r}\text { Palladium } 41,8 \mathrm{mg} \\
=3,6 \mathrm{cmm}\end{array}$ & 114 & $\$ 60$ & 266 & \multirow{2}{*}{\multicolumn{2}{|c|}{125}} & \\
\hline Palladiun $111 \mathrm{mg}$ & $-\ldots$ & 737 & 248 & & & \\
\hline Palladium 89 ing & -- & 800 & & hd im G & ebläse ans & egl. \\
\hline
\end{tabular}

Aus dieser Tabelle ist ersichtlich, dass Palladium in gewöhnlichem Zustande ca. das 110 fache seines eigenen Volumens an Gasen occludirt enthält, die erst durch längere Rothgluth im Vacuum frei gemucht werden können. Wird Palladium, das alle occludirten Gase abgegeben hat, von neuem mit einem Gase zusammengebracht, so occluảirt es dieses sofort wieder, und waren die gefundenen Unterschiede bei Kohlenoxydgas, Sauerstoff und Luft nur gering. Die Wasserstoffocclusion ist sehr bedeutend, und ist die Grösse derselben ebenfalls wie beim Platin davon abhängig, ob das Metall vollständig gasfrei war oder aber noch andere Gase occludirt enthielt.

$\$ 19$. Aus den beschriebenen Occlusionsversuchen ersieht man, dass bei Palladium und Platin die Wasserstoffocclusion durch eine unreine Oberfläche sehr gestört wird und nur bei vollkommen reiner, metallischer Oberfäache eine Wasserstoffocclusion bei gewöhnlicher Temperatur eintritt. und zwar ist dann die Wirkung des Palladiums ca. 4 wal so stark als bei Platin. Greifen wir jetzt zurück auf die katalytische Wirkung dieser Metalle und erinnern uns, dass dieselben die Katalyse bei gewöhnlicher Temperatur nur dann 
zeigen, weun sie eine reine metallische Oberfläche haben, so finden wir die vollständige Uebereinstimmung in den Bedingungen zur Katalyse und Wasserstoffocclusion bei niedriger Temperatur. Es ist, wenn beide Erscheinungen im $\mathrm{Zu}$ sammenhang stehen, zu erwarten, dass Palladium mit reiner Oberfläche bei Zimmertemperatur viel stärker katalytisch wirkt als Platin. Da dieser Versuch noch nicht gemacht ist. und ich auch nirgends Angaben über die Grösse der kata. lytischen Wirkung des Palladium bei niedriger Temperatur gefunden habe, so wurde dieser Versuch an dieser Stelle nachgeholt und gefunden, dass dissselbe bedeutend stärker wirkt als Platin. Der Versuch wurde so angestellt, dass je ein Streifen ron Palladiumblech und Platinblech nach Faraday mittelst des galvanischen Stromes gereinigt und hierauf in ein über Wasser abgeschlossenes Knallgasvolumen gebracht, wurde. Obgleich beide Bleche bei gleicher Dicke -- cr. ${ }^{1} / 10 \mathrm{~mm}$ - ungefähr gleiche Oberflüche hatten, waren durch das Palladium in 30 Minuten 19 ccm Knallgas in Wasser verwandelt, während das Platin in derselben Zeit nur $4 \mathrm{ccm}$ chemisch verbunden hatte.

$\S 20$. Ich glaube nicht fehl zu gehen, wenn ich in Rücksicht auf die Resultate meiner Versuche annehme, dass die katalytische Wirkung der Metalle die Wirkung des von denselben occludirten $W$ asserstoff's ist, welcher wahrscheinlich in allen Metallen als nascirender Wasserstoff wirkt. In der That wurde dies letztere für den von Palladium oceludirten Wasserstoff durch Versuche von Graham ${ }^{1}$ ) schon lange nachgewiesen, und die Annahme eines analogen Verhaltens der übrigen Metallwasserstofiverbindungen ist, soweit mir bekannt, noch nicht durch Versuche widerlegt worden.

Die Katalyse bei hoher Temperatur in den $\$ 6$ beschriebenen Versuchen, bei welchen die Metallobertläcbe nicht rein war, können wir uns nur auf gleiche Weise erklären, wie bei niedriger, d. h. als Wirkung des occludirten Wasserstoffs. Wir müssen daher annehmen, dass die hohe Temperatur die

1) Grabum, Phil. Miag. (4) 32. p. 503. 1. B. 49. 1866. 
Wasserstoffocclusion begünstige, und zwar in der Weise, dass eine dünne Schicht fremder Stoffe, welche das Metall bedeckt, bei hoher Temperatur die Occlusion weniger als bei niedriger stört. In der That hat Graham bei seinen Versuchen gefunden, dass die grössten Gasmengen occludirt werden, wenn er die Metalle stark erwärmte und alsdann in der Gasatmosphäre erkalten liess.

Phys. Lab. der Unir. Freiburg i. B., im Juni 1888.

\section{Drei neue Methoden zur Bestimmung der magnetischen Inclination; von Carl Ludwig Weber. \\ (Hierun Taf. VI Fig. 9.)}

Während zur Bestimmung der Horizontalintensität des Erdmagnetismus eine grosse Zahl von verschiedenen Methoden existirt, war die Messung der Verticalcomponente, resp. der Inclination bis vor kurzem fast ausschliesslich auf die Anwendung des Inclinatoriums und des Erdinductors beschränkt. Erst in den letzten Jahren sind mehrere Vorschläge zur Abänderung oder Ersetzung dieser beiden Bestimmungsweisen hervorgetreten ${ }^{1}$, die zu beweisen scheinen, dass ein Bedürfniss vorliegt, neue Wege zur Bestimmung der Inclination zu erschliessen.

Das Folgende enthält drei Methoden, welche das angedeutete Ziel auf einem bis jetzt noch nicht eingeschlagenen Wege zu erreichen suchen; die Beschreibung eines dabei verwendeten Instrumentes und die vorläufigen Resultate, welche nach einer der drei Methoden erhalten wurden. Diese letzteren, mit einem noch sehr unvollkommenen Instrumente erhalten, besitzen noch nicht jene Genauigkeit, die mit den

1) K. Schering, Gött. Nachr. 1882. p. 345; Wild, Exner's Rep. 18. p. 111. 1882; Riecke, Wied. Ann. 13. p. 198. 1881; Krüger, Wied. Any. 28. p. 612. 1886; Liznar, Exner's Rep. 23. p 306. 1887. 